\title{
"Un feminismo nominal". Victoria Ocampo y las importaciones fragmentarias. Una lectura de José Amícola (2019). El poder-femme. Virginia Woolf, Simone de Beauvoir y Victoria Ocampo. La Plata: Edulp
}

\author{
Malena Botto \\ malena_botto@yahoo.com.ar \\ Universidad Nacional de La Plata-Universidad \\ Pedagógica Nacional, Argentina
}

Recepción: 18 Diciembre 2019

Aprobación: 06 Septiembre 2020

Publicación: 01 Marzo 2021

\begin{abstract}
Cita sugerida: Botto, M. (2021). “Un feminismo nominal”. Victoria Ocampo y las importaciones fragmentarias [Revisión del libro El poder-femme. Virginia Woolf, Simone de Beauvoir y Victoria Ocampo por J. Amícola]. Descentrada, 5(1), e142. https://doi.org/10.24215/25457284e142
\end{abstract}

\section{UN LIBRO ACCESIBLE; UNA REVISIÓN A LOS ESTUDIOS SOBRE EL CAMPO LITERARIO}

En primer lugar, me interesa señalar esa particularidad del libro de José Amícola. Es un escrito que de una manera lúcida toma a las figuras de Virginia Woolf, Simone de Beauvoir y Victoria Ocampo, y las ve no simplemente en sus relaciones (o no relaciones) personales, sino participando o liderando formaciones culturales que también se deben poner en diálogo, en una consideración que toma en cuenta tanto las disputas y tensiones en los ámbitos locales, como los "vasos comunicantes" (Amícola, 2019, p. 14) que se dan entre esas comunidades interpretativas, con sus inclusiones y exclusiones. Fundamentalmente, en tanto detrás de la anécdota de tal o cual episodio, de una carta dirigida de una a la otra, o de una visita, siempre se trasluce o se señala explícitamente el modo en que estas relaciones están atravesadas por la marca de lo geopolítico, de las nociones de centro y periferia y de desigualdades en cuanto al lugar de enunciación de cada quien.

Estas cuestiones que también hacen a los estudios del campo son parte de lo que Bourdieu nollegó a trabajar en sus teorizaciones y, hoy por hoy, podemos decir que es una parte fundamental, retomada por investigadores formados por él, como Gisèle Sapiro. Me parece por ende muy valiosa la manera como Amícola reconstruye 
este entramado de relaciones, tensiones y exclusiones, y lo hace, no para el caso de la literatura más actual, sino trabajando con formaciones culturales de peso durante la primera mitad o mediados del siglo XX.

Además, por supuesto, del aporte que brinda lo que constituye el eje central del estudio, es decir, la consideración del sistema-género, ofrece una articulación que, hasta donde conozco, no solo no ha sido trabajada por Bourdieu en sus estudios del campo literario, sino que tampoco ha sido incorporada por sus discípulos o seguidores (como sí la transnacionalización de las obras, la consideración de los nuevos soportes y tecnologías de la cultura digital, etc.).

Si bien el autor no trabaja de modo explícito con la idea de campo de Bourdieu o con la noción de formaciones de Raymond Williams -sino que habla de comunidades interpretativas excluyentes- creo que son nociones que gravitan lo suficiente como para que se considere la particular perspectiva que el autor utiliza, y su combinatoria con el estudio del sistema-género, como un aporte significativo a los estudios antes mencionados, no solo o exclusivamente a los estudios de género.

Pero, además, lo que me interesa señalar especialmente, es que el libro hace todo eso de una manera y con un tono que es muy amable para con el lector. Quienes hemos leído otros estudios del autor estamos acostumbrados a un estilo que conjuga el dato erudito, las traducciones propias desde diversos idiomas, las referencias teóricas y también etimológicas, junto con la anécdota en apariencia trivial pero ilustrativa de lo que se discute, las rencillas cuasi domésticas, en fin, un conjunto que da cuenta de una serie sumamente extensa de búsquedas y hallazgos. Y que todo eso se conjugue en un estudio "de divulgación”, en el mejor de los sentidos del término, que consigue no dejar afuera al lector no especializado. En este nuevo libro creo que se exacerba esta característica, lo cual no es poco decir.

\section{VICTORIA OCAMPO: UNA RELECTURA}

Dicho esto en términos generales, y para volver también a la cuestión de los diferentes lugares de enunciación y la cuestión geopolítica, de las tres figuras femeninas que constituyen el estudio me voy a detener brevemente en la de Victoria Ocampo, no tanto porque sea la figura argentina, sino porque me parece que la puesta en primer plano de la cuestión del sistema-género y ciertos modos personales de interpretación le permiten a Amícola sopesar lo que representa esta escritora y en general el legado de Sur, sobre el que tanto se ha escrito, de una manera también particular y con aristas novedosas.

En una primera caracterización se presenta a Ocampo como: "Dueña por derecho propio de toda la impronta de las clases dominantes en la Argentina agro-exportadora” (Amícola, 2019, p. 100). Su nombre mismo (Victoria) tiende un puente con el Imperio Británico, en aquella época de "relaciones carnales" que es la década infame de 1930. El autor va a sugerir, a lo largo de su análisis, que no es solo el nombre lo que en Ocampo lleva la impronta victoriana, en contraste con la figura de Virginia Woolf -tan admirada por Victoria- y con todo el grupo de Bloomsbur y, que precisamente reaccionan contra todos los resabios victorianos que persistían en la cultura y la sociedad inglesas de los años 20 y 30 . Voy a volver al final sobre esta relación entre Victoria y Virginia, y sobre los modos de la conciencia feminista en cada una de estas escritoras.

Me interesa destacar algunas cuestiones que hacen a la revista Sur, que Amícola atribuye en buena medida a la personalidad, a los caprichos o a las limitaciones ideológicas de Victoria Ocampo, y que imprimen una particularidad al estudio.

En primer lugar -y esto atraviesa todo el análisis-, si se suele afirmar repetidamente que Victoria Ocampo fue una liberal conservadora que, en última instancia, respondió a los intereses de su clase antes que a reivindicaciones progresistas de distinta índole, es también común que su feminismo aparezca como una excepción o salvaguarda, como si pudiera haber desarrollado un coto feminista dentro de su personalidad que quedase a salvo de sus posiciones conservadoras o incluso reaccionarias en otros ámbitos y frente a otros fenómenos. Amícola señala, y lo documenta a través de numerosos ejemplos de sus escritos y de episodios de 
su vida -así como de sus decisiones editoriales en Sur-que en definitiva esa coexistencia tan contradictoria no es posible, y que el feminismo de Ocampo es meramente declarativo.

En la década del ' 30 esto va a aparecer ejemplificado no solo por la influencia arrobadora que ejerce en ella la figura de Eduardo Mallea, sino también por el poco tino con el que, a juicio del autor, Ocampo en tanto operadora cultural al frente de Sur promociona en Buenos Aires las conferencias del Conde de Keyserling, cuyo irracionalismo deriva en una metafísica que le permite ponerse a sí mismo y su propia vida como modelo ontológico, primer representante del superhombre nietzscheano o de la nueva humanidad. De esta influencia surgen, señala Amícola, ciertos ensayos fatalistas de Mallea, así como ciertas ideas en torno a la "primacía de la sangre", que Ocampo luego descartará pero que en este momento la seducen. Podría considerarse que el feminismo de la directora de Sur en este momento se encuentra todavía "en ciernes" (Amícola, 2019, p. 119). No obstante, todo el análisis que el autor realiza respecto de la relación de Sur con el existencialismo durante los años 40 y la posición de Ocampo en torno al binomio Sartre-Beauvoir, va a dejar en claro que ese argumento no termina de convencer.

\section{El existencialismo en Sur}

Victoria Ocampo no simpatizaba con Sartre, y de ello es testimonio la escasa difusión de sus ideas en la revista Sur. Las inclusiones se deben a las participaciones de Ernesto Sábato y, sobre todo, de José Bianco, quien para Amícola -apoyándose también en la lectura de Rosalie Sitman (2003)- es quien logra sostener la hegemonía de Sur durante toda la década de 1940, en su labor de Secretario de Redacción (había reemplazado a Mallea en este puesto, desde 1938). No obstante, Victoria Ocampo no comparte en términos generales sus posiciones y va a terminar por imponerse, para luego romper relaciones con Bianco, cuando éste acepte ir a Cuba como jurado del Premio Casa de las Américas, en 1961.

La directora, en cambio, va a dar una preeminencia indiscutible en las páginas de Sur a la figura de Albert Camus, quien, al menos en apariencia, encajaba perfectamente con la noción de "justo medio", absolutamente liberal, que Ocampo pretendía sostener a ultranza como una forma de mantenerse "a salvo de partidismos" (Amícola, 2019, p. 103). En ese sentido, tanto Sartre como su compañera Simone de Beauvoir van a representar en el campo francés de posguerra el "ala dura" del comunismo, y en otro capítulo del libro se reseñan con detalle las disputas que Camus -en tanto excluido del círculo del Café de Flore- va a sostener hasta su muerte, en 1960. En tanto caja de resonancia implícita de estas disputas que se dan en el continente europeo, Sur y Victoria Ocampo optarán por la figura de Camus, un individualista liberal que durante la posguerra se había quejado de que los alemanes y la cuestión de la ocupación nazi habían pretendido "hacerlo entrar en la Historia”. De igual modo, Camus reprochará luego a Sartre su silencio frente a los crímenes del estalinismo, sin disponerse él mismo a denunciar, para el caso de Argelia, las torturas perpetradas sobre los ciudadanos árabes en la colonia.

Por otro lado, El hombre rebelde -ensayo de Camus de 1951 que genera toda una polémica en las páginas de Les temps modernes- toma del filósofo alemán Max Scheler una distinción algo caprichosa entre las nociones de "rebeldía" y "resentimiento", proponiendo un concepto de hombre (no de mujer) "rebelde", en el sentido de "disconforme con el sistema" (en contraste con la etimología francesa, que supone para "rebelde" la misma raíz de "revolucionario"). Esa operación permite a Camus meter en la misma bolsa un espectro de personalidades que van desde el Marqués de Sade a Hitler y a Stalin, pasando por Rimbaud.

En suma, esta toma de partido por Camus por parte de Victoria Ocampo no solo redunda en que las referencias al existencialismo en Sur acaben siendo "vagas y erráticas", como señalara Nora Pasternac (2002), sino que, para Amícola, implica también que a través de la pluma del existencialista heterodoxo se colara en Sur una "misoginia decontrabando", sin que Victoria Ocampo (aparentemente) lo advirtiera.

En efecto, Camus va a ser refractario al feminismo, pero, además y sobre todo, va a acabar decididamente enfrentado con Simone de Beauvoir, a quien le cuadraría el mote de "resentida" de acuerdo con la clasificación 
recién comentada. Y, en este punto, es difícil decir que Victoria Ocampo pudiera ignorar la cuestión. Simplemente, compartía la apreciación de Camus. Aún más: hay en la directora de Sur una ceguera impuesta por su procedencia de clase, y resulta sumamente ilustrativo para entender su apreciación de la intelectual francesa un pasaje rescatado por Amícola. Escribe Ocampo en una carta de 1963 desde París, mientras leía las memorias de la aristócrata Ottoline Morrell, que éstas le gustan sin duda más que las de Simone de Beauvoir, dado el "intransigente comunismo" de quien cataloga como "esta maestrita con mentalidad de resentida de clase media (...) y complejo de superioridad" por haberse acostado con el "inteligentísimo y físicamente repelente" autor de A puertas cerradas (Amícola, 2019, p. 129).

El derrotero del existencialismo en Sur, y también este tipo de posicionamientos y consideraciones por parte de su directora, hacen a la decadencia de la revista en el sistema literario argentino a partir de los años 50, de una manera que, según Amícola, no ha sido suficientemente señalada, como sí lo han sido su rechazo tajante a una reconsideración del peronismo, tal como lo hizo Contorno, su posición intransigente respecto de la Revolución Cubana y la insistencia en separar -a la vez que constante e implícitamente mezclabanliteratura y política. Esos factores hacen que la formación cultural de cuño liberal que conforma Sur aparezca como cada vez más anacrónica y fuera de contexto.

No obstante, José Amícola destaca, en tanto factor de decadencia, no solo esta fragmentaria recuperación del existencialismo (aquí nuevamente Sur se opone a Contorno) sino, además, su rechazo del psicoanálisis freudiano -tan pregnante en Buenos Aires desde la década del '30- como luego de las teorías lacanianas. En materia de psicoanálisis, Sur recupera la figura de un irracionalista como Carl Jung. El binomio Camus -Jung frente a Sartre-Freud o luego Lacan aparece como la opción por dos europeos que fueron heterodoxos en lo suyo y discutidos en sus círculos.

Finalmente, también hay que tener en cuenta ciertas posiciones de Ocampo en el campo artístico que resultan cuasi reaccionarias, como el rechazo del teatro moderno de Tennessee Williams o Jean Genet, del surrealismo (tanto el francés como el movimiento argentino de los años 40). Se trata de una postura que se vuelve cada vez más refractaria a las manifestaciones de vanguardia, que Victoria Ocampo sí había sabido apreciar en sus inicios, cuando viajaba por Europa en su juventud. La gravitación de la figura de un "clasicista" como Paul Valéry en la revista Sur es otra muestra de ello.

Tanto la ceguera ideológica atribuible a su origen de clase como el planteo de una política de la literatura que además de europeizante resulta demodé, lleva al autor del estudio a considerar que: "Estos errores del supuesto olfato infalible de Victoria Ocampo [en tanto operadora cultural] son para mí el mejor de los ejemplos de su rigidez mental, que solo puede ser comprensible si también se analiza su snobismo" (Amícola, 2019, p. 114).

Y en este último punto -el del snobismo- es interesante el rescate que hace Amícola de un estudio temprano como es el de Blas Matamoro (1986), y el señalamiento acerca de cómo ese crítico supo leer mejor que otros, desde la perspectiva del sistema-género, lo que suponen las reivindicaciones feministas de Victoria Ocampo, cuyo feminismo podría calificarse, y así lo hace Amícola, como "nominal".

Matamoro, por su parte, refiere a los "espejos victoriales": una serie de personalidades masculinas que la directora de Sur eligió para que le sirvieran como modelo. Entre ellas se cuentan el ya mencionado Conde de Keyserling, José Ortega y Gasset y, por supuesto, Eduardo Mallea. Todos ellos, entendidos por Amícola como "machos depredadores", le devolvían a Victoria Ocampo una imagen de "hembra sudamericana”, y un rol específico en el que la elegancia y la belleza resultaban valoradas por encima de la inteligencia; rol que a ella jamás se le ocurrió cuestionar, sino que aceptó plenamente.

Entonces, junto a la cuestión de clase que la lleva a rechazar a Simone de Beauvoir y otras formas de "rigidez mental" propias de una educación conservadora, aparece este feminismo nominal, propio de una forma de snobismo (es decir, una vez más, de imitación de modelos europeos leídos muy fragmentariamente). 


\section{La RELACión de Victoria Ocampo con Virginia Woolf}

En ese sentido, vuelvo brevemente a la figura de Virginia Woolf mencionada antes, por quien Victoria sentía una fascinación que, luego de considerar todo lo expuesto, aparece como disonante. Amícola señala justamente eso, cuando en general se la interpreta como una consecuencia "natural" del "feminismo" de Ocampo.

Si bien su formación en la consideración de la cultura inglesa como modélica y el origen de clase de Virginia Woolf explicarían una simpatía que Victoria Ocampo no hubiera podido profesar jamás a Simone de Beauvoir, queda clarísimo después de leer este estudio hasta qué punto Victoria Ocampo realiza una interpretación sesgada de lo que representa la tan admirada figura de Woolf. Su consideración omite cuestiones "tabúes" para la argentina, como la declarada bisexualidad de la escritora inglesa y, en general, la defensa de las libertades sexuales que el grupo de Bloomsbury reivindicó como un modo especialmente sensible de rechazo a la moral victoriana (si detrás de esa apreciación tan sesgada como idealizada se esconde una atracción homoerótica de Victoria hacia Virginia, es algo que solo puede sugerirse, dirá el autor).

En todo caso, José Amícola sí deja planteado que esta fascinación "puede pensarse como un gran malentendido entre ambas personalidades, pues cada una vio lo que quería ver en la otra” (2019, p. 122). Para el caso de Virginia Woolf, podemos suponer que se trataría no solo de pasar por alto los ya mencionados "errores en el olfato cultural" de Ocampo, sino también el hecho de que, cabalmente compenetrada en su rol de "hembra sudamericana", Victoria Ocampo se presentara a visitarla llevando como obsequio una colección exótica de alas de mariposa, provenientes de los países latinoamericanos, cuya cultura la directora de Sur se empeñó en ignorar olímpicamente.

Parte de este gran malentendido lo constituiría el hecho de que Victoria Ocampo haya revisado y dejado pasar las desafortunadas decisiones que aparecen perpetradas por "el clan Borges" (sea madre, padre o hijo) ${ }^{1}$ en la traducción de Orlando realizada para Sur en 1938, y que es comentada en el libro en el capítulo del libro dedicado a Bloomsbury. En la lectura que hace José Amícola, al dejar que prevaleciera la fascinación antirrealista por la biografía imaginaria -a la que Borges dio preeminencia en la traducción, y que el grupo de Sur comparte por esos años- y permitir con ello que aparecieran silenciadas o asordinadas las potentes desestabilizaciones al sistema-género representadas en la androginia del personaje, a Ocampo nuevamente parece escapársele la tortuga de las reivindicaciones feministas, y del lugar propio que adquiere la escritura de Woolf en virtud de esta característica. Puntualmente, el autor del estudio se refiere al episodio clave de transformación del personaje, su devenir-mujer. Si en el inglés original la escena aparece signada por la mención de "trompetas" y "trompeteros" -todos símbolos fálicos que se reúnen para saludar la presencia del no-falo-, la traducción firmada por Borges solo refiere a "trompetas". Más importante todavía: cuando culmina el episodio, el narrador de Woolf señala "He was a woman" ("Élera una mujer”), en tanto Borges traduce: "Era una mujer". La omisión del pronombre no puede explicarse simplemente por el hecho de que, en términos gramaticales, el español no lo exija, en tanto el inglés sí lo requiere, sino que debe leerse en sentido ideológico, como un borramiento de las marcas de género (Amícola, 2019, pp. 53-60).

\section{¿UNA HEREDERA?}

Como consideración final en torno a la semblanza de Victoria Ocampo que aparece en este estudio, no puedo dejar de señalar algo que en la lectura me resultó muy sugerente. Hacia el final del libro, se retoma la idea de que Victoria Ocampo no fue capaz de inspirarse en modelos rioplatenses -como por ejemplo Juana Manuela Gorriti- por considerarse "adosada al vacío”, como le había expresado a Virginia Woolf en una carta. Y se señala que tampoco, dada la tradición clasista y liberal a ultranza que representó, pudo tener sucesoras. "Salvo -dice Amícola- que se considere a Beatriz Sarlo como su discípula" (2019, p. 246). Esta reflexión, 
que no tiene la forma de una afirmación tajante sino el encanto de la sugerencia, me parece no solo original sino sumamente potente para plantear o continuar la discusión, y una muestra más de hasta qué punto el foco puesto en la discusión del sistema-género permite en este trabajo una discusión mucho más amplia que hace a la historización de las configuraciones y los posicionamientos dentro del campo literario, con especial consideración de las opciones político-ideológicas.

\section{REFERENCIAS}

Amícola, J. (2019). Elpoder-femme. Virginia Woolf, Simone de Beauvoir y Victoria Ocampo. La Plata: Edulp.

Matamoro, B. (1986). Genio y figura de Victoria Ocampo. Buenos Aires: Eudeba.

Pasternac, N. (2002). Sur: una revista en la tormenta. Los años de formación (1931-1944). Buenos Aires: Paradiso.

Sitman, R. (2003). Victoria Ocampo y Sur: entre Europa y América. Universidad de Tel-Aviv: Instituto de Historia y Cultura de América Latina.

\section{Notas}

1 Amícola explica que la traducción de Orlando (1928) fue encargada a Borges por Victoria Ocampo, y es la que efectivamente lleva la firma del escritor y que ha resultado tan prestigiada en todo el ámbito latinoamericano. No obstante, existen sospechas -nunca confirmadas ni descartadas- de que la labor de traducción haya estado en verdad a cargo de su madre, Leonor Acevedo de Borges, o -en otras versiones- de su padre, Guillermo Jorge Borges. El autor de $E l$ poder-ferme... ironiza sobre este "misterio", refiriéndose a la traducción de Sur como la del "clan borgeano" (Amícola, 2019, p. 56). 